\title{
PROGRESS TOWARD SHOCR ENHANCEMENT OF SUPERSONIC COMBUSTION PROCESSES
}

\author{
Frank E. Marble*, Gavin J. Hendricks** and Edward E. Zukosk i* \\ California Institute of Technology
}

\section{ABSTRACI}

In air breathing propulsion systems for flight at Mach numbers 7 to 20 , it is generally accepted that the combustion processes will be carried out at supersonic velocities with respect to the engine. The resulting brief residence time places a premium on rapid mixing of the fuel and air. To address this issue we are investigating a mechan ism for enhancing the rate of mixing between $a$ ir and hydrogen fuel over rates that are expected in shear layers and jets.

The mechanism rests upon the strong vorticity induced at the interface between a light and heavy gas by an intense pressure gradient. The specific phenomenon under investigation is the rapid mixing induced by interaction of a weak oblique shock with a cylindrical jet of hydrogen embedded in air. The status of our investigations is described in three parts: a) shock tube investigation of the distortion and mixing induced by shock waves impinging on cylindric of hydrogen embedded in air, b) the molecular mixing and chemical reaction in large vortices, periodically formed in a channel, and c) two-dimensional non-steady and three-dimensional steady numerical studies of shock interaction with cylindrical volumes of hydrogen in air.

\footnotetext{
* Professor, Mechanical Engineering and Jet Propulsion

** Postdoctoral Fellow, Jet Propulsion
} 


\section{INTRODUCTION}

Flight at hypersonic Mach numbers, between 6 and 15, appears as the next regime of major aeronautical research and development. The "trans-atmospheric aircraft," having a major component of airbreathing propulsion together with the capability of taking off from a runway and climbing to low earth orbit, is perbaps the most ambitious example. The operational flexibility of such an aircraft could offer a substantial advantage over current systems. The actual advantage that may be realized can be determined only by detailed investigations of the aerodynamic, cooling and propulsion problems which are unique to this Mach number range and by rather sophisticated studies of integration between the aerodynamic configuration, the propulsion system and the thermal control of the structure. The configuration and integration analyses which appear in Kirkham \& Bunt (1977), Edwards, Smal1 \& Weidner (1975), Small, Weidner \& Johnst on (1974) and Nowak \& Relley (1976) provide substantial insight into both the importance and the formidable difficulty of the problem.

Among the conclusions of these and further studies is the clear confirmation that the problem of efficient, compact combustion stands as a central issue. In the first place, because of the severe penalties associated with excessive $108 s e s$ and hesvy beat load, it is generally accepted that the combustion process must be carried out in a gas stream that is supersonic with respect to the engine. As a consequence, there is a very short time available for mixing and reaction and a high probability for internal shocks and losses. Because of the very high free-stream stagnation temperature associated with hypersonic velocities, the relative stagnation temperature rise resulting from combustion is rather smal1. Under this circumstance, internal aerodynamic losses or incomplete chemical reaction results in an unacceptable deterioration of performance.

In order to achieve a compact combustion process, considerable effort, e.8. Gross (1959), Gross \& Oppenheim (1959) was devoted toward combustion in stabilized detonation waves. The results were not encouraging. Studies directed toward rapid mixing, Ferri (1968), Ferri (1973), Evans \& Anderson (1974), McClinton (1978), have resulted in greater losses and longer combustors than desirable. Recent excellent reviews, Waltrup (1986) and Northam \& Anderson (1986), report the current status of the combustion studies and confirm that the problem is still an open one, particularly at Mach numbers greater than 7. Therefore it is appropriate to investigate means for enhancing the rate of mixing between air and hydrogen fuel over that which may be achieved in shear layers and jets.

The mechanisu to be described here depends essentially upon the Rayleigh-Taylor instability induced at the interface between a light and heavy gas by a strong pressure gradient. It is a property of combustion fields that the distribution of gas density is exceedingly non-uniform and this characteristic is accentuated when the fuel is hydrogen. One of the major effects of very strong pressure gradients on a field of non-uniform gas density is to accelerate the lighter gas at a rate several times that of the heavy $8 a s$. In addition to generating vorticity in the region of high density gradient, the large 
scale stability of the field is affected and flow reversal may be induced, a phenomenon which is of paramount importance in the supersonic mixing and combustion of hydrogen in air.

In an actual supersonic combustor there will exist oblique shock waves which constitute highly concentrated forms of the adverse pressure gradients discussed above. It is to be expected that the flow reversal, vortex formation and mixing observed in the steady phenomenon will appear, but in a more intense form. Therefore the distinct possibility exists of utilizing a set of "tailored" steady or unsteady shocks to induce very high intensity mixing and chemical reaction between the air and hydrogen fuel.

We are at present in the early stages of an extensive experimental program to investigate the detailed mechanism of the shock induced enhancement of mixing in non-uniform gas streams. The technological process described above has been broken down into two relatively independent phases for detailed experimental investigation, the first of which is being carried out in the Galcit 17-inch shock tube, the second in our unsteady combustion facility.

The work described here is supported by the United States Air Force Office of Scientific Research under Grant Number AFOSR-84-0286 and under URI Grant F49260-86-C-0113. Both programs are under the direction of Dr. Julian M. Tishkoff. 


\section{THE ENHANCEMENT MECHANISM AND BACRGROUND INFORMATION}

When the pressure gradient in a gas is not aligned with the density gradient, vorticity may be generated in the gas. If the gas undergoes an isentropic change of state, the direct relationship between the pressure and the density guarantees the colinearity of these two gradients and no vorticity is generated. When, however, the density gradient is in part due to a variation of gas composition or the non-uniform conversion of chemical to sensible heat, the two gradients will not be aligned. Then, as shown in Fig.l, the displacement of the mass center from the geometric center of a gas element will allow the normal pressure stress on the surface of the element to generate a moment about the mass center and, hence, a rotation. A convenient analytical statement of this phenomenon is contained in Bjerknes theorem

$$
\frac{d \Gamma}{d t} \cdot \int \frac{1}{\rho^{2}}\{\operatorname{grad} \rho \times \operatorname{grad} p\} \cdot d A
$$

where $\Gamma$ is the circulation about a closed contour fixed to the fluid.

As applied to combustion problems, this vorticity generation mechanism was investigated explicitly by Fleming (1982) and that type of analysis was extended to relatively strong adverse pressure gradients by Marble \& Hendricks (1986a). In this study a diffusion flame was situated, $F$ is. 2 , along the horizontal axis and the gas flowed against a rising pressure field. The combustion products, which were of relatively low density, could not easily move against the pressure gradient and, as a consequence, reversed their flow direction and moved back upstream. The velocity and temperature profiles for a particular pressure gradient are shown in Fig. 3. In this circumstance the pressure gradient and the density gradient were nearly normal to each other, yielding the maximum rate of vorticity generation. This vorticity appears in the shear layers that bound the region of backflow, Fig. 3 .

Consider now a cylindrical mass of low density gas (e.g. hydrogen) embedded in a higher molecular weight gas (e.g. air) situated in a pressure gradient as shown in Fig. 4a. The density jump between the hydrogen and the air constitutes a strong density gradient and this interacts with the imposed pressure gradient, according to Bjerknes theorem, to produce the vorticity distribution shown in Fig. $4 \mathrm{~b}$ at the boundary of the bydrogen mass. The velocity field induced by the vorticity at the interface, distorts the boundary of the hydrogen mass, as shown schematically in Fig. 4c, and essentially creates a strong vortex pair.

Consider finally a shock wave passing over the cylindrical mass of bydrogen; this, as mentioned earlier, constitutes a very concentrated form of adverse pressure gradient. The shock passage, which can be considered very fast in comparison with other processes, deposits a distribution of vorticity over the boundary similar, but generally much stronger, than that associated with usual cont inuous gradients. Some of the earliest observations related to this 
phenomenon, reported by Rudinger (1958), Rudinger \& Somers (1960), concerned the passage of a shock wave over bubbles and over flame surfaces. Subsequent computations by Evans et al (1962), Picone \& Boris (1983) and Picone et al (1983) demonstrated the possibility of calculating the deformed surfaces observed by Rudinger (1958) with a relatively high degree of accuracy.

The earliest experiments which are directly related to the present investigations were performed by Sturtevant together with his graduate student, Hass (1983). These were shock tube studies, carried out at Caltech, concerning the interaction of relatively weak shock waves with regions of low density gas embedded in high density gas. The research was primarily aimed at the problem of shock diffraction in non-uniform atmospheres; fortunately the photographic data recording was carried to more than a millisecond after the shock impact and, as a consequence, preserved information valuable to the present investigation.

This interaction was found to generate a strong vortical motion, originating along the interface between the two gases. Severe and rapid distortion of the light gas region was observed followed by intense mixing. Figure 5 consists of three shadowgraphs taken during the passage of a Mach 1.22 shock wave over a $5 \mathrm{~cm}$ diameter cylinder helium embedded in air. The time elapsed after the shock impinges on the helium cylinder is given in milliseconds for each photograph. The results show, in a most striking manner, the generation of a vortex pair by the vorticity generated at the initial gaseous interface and the subsequent diffusion of the vorticity from that surface. This provides the time dependent development of the flow field described earlier in this section. The shock tube experiment does not, of course, include the density change associated with combustion, a factor which would further increase the intensity of the induced flow. 


\section{ANALYTICAL AND COMPUTATIONAL STUDIES}

Referring specifically to the experiments shown in Fis. 5 the phenomenon may be divided, conceptually, into two phases. In the first phase, the vorticity is deposited at the interface between the helium and $a$ ir and the ensuing motion generates an intense vortex pair. In the second phase these vortices entrain air into the helium and undergo a complex mixing process, leading to molecular mixing and chemical reaction. Although some of the results obtained by Marble (1985), Raragozian \& Marble (1986), Raragozian \& Manda (1987) are of interest with respect to the second phase, the discussion here will concentrate on the analysis of the initial distortion of the light gas region.

During the past few months we have carried out Euler code calculations, Marble \& Hendricks (1986b), to investigate the distortion of a cylindrical mass of helium embedded in air, by the impingement of shock waves of various strengths. The code employed was one originally developed by Eric Baum of the Electronics and Defense Sector, TRW, for use in the analysis of shock tube experiments. We have modified the original code considerably and adapted it for the present calculations in non-uniform'gases.

It is of interest first to examine the time period over which we are able to obtain a ressonable representation of the distorted belium cylinder observed in the experiments mentioned in the previous section, shown in Fig. 5; the photos were taken at times of 132,378 , and 724 microseconds respectively after the initial impingement of the shock on the helium. The wire hoop which appears in the photographs is one of those that supported the microfilm sheath which contained the helium before shock impingement. It is reasonable to assume that the wake of the ring support, particularly visible in $F$ ig. $5 \mathrm{~b}$, is locallized at the ends of the helium mass and has a small effect upon the larger portion of the gas into which the camera is looking. The boundary of the helium remains fairly well defined through this time span.

A reasonable test of our Euler code then is to make calculations of the field quantities at similar times and to compare the gas boundary contours. Three of these computed shapes, at comparable times after the shock impact are shown in Fig. $6 a, 6 b$, and $6 c$. The boundaries of these computed contours tend to diffuse somewhat with time and these thicker boundaries have been reduced on the contours shown. Clearly the comparison between computed and experimental boundary shapes is adequate to assure us that other computed properties are correspondingly accurate. These will be presented subsequent ly. 
Return for the moment to the technological issue of how such mixing augmentation could be employed if indeed the present program demonstrated that its use was warranted. Consider a single jet of hydrogen moving with a supersonic velocity parallel to the supersonic airstream toward or within the engine. Let this jet be intersected by an oblique shock wave, as shown schematically in Fig. 7. Then in eacb succeeding cross section of the jet, the shock appears moving across the jet in the manner suggested by the sketches on the figure. To the extent that this problem can be analyzed by conventional "thin body" theory, $x / 0$ can be exchanged with time in the unsteady calculations and the results applied accordingly. Thus the jet should develop a strong vortex pair parallel with the direction of flow as the oblique shock intersects it and will mix rapidly downstream of the shock.

The accuracy with which this thin body theory may be applied has been examined numerically by considering the steady problem of an oblique shock intersecting a cylindrical (non mixing) co-flowing jet of bydrogen. The results in successive cross sectional planes will be compared with the corresponding two-dimensional calculations.

Figure 8 shows the distortion of the jet when the shock intersection has passed to the point indicated. The two plots below the distorted jet contour give respectively the corresponding density and vorticity distributions. Notice particularly the distribution of vorticity building up strongly on the portions of the interface nearly normal to the shock wave, as suggested by the vorticity generation mechan ism described earlier. Figure 9 provides similar information for a section further downstream along the jet, beyond the point where the shock passes out of the jet. Here the complete vorticity distribution is developed and it provides a very clear intuitive picture of how the jet distortion is induced.

Figures 10 and 11 present corresponding information computed from the two-dimensional unsteady model. The correspondence is extremely close, even in small details of the density distribution. It is possible, of course, that the comparisons will be less favorable for lower jet Mach numbers, but the differences should not be great so long as the hydrogen jet is supersonic.

This result is of great consequence with regard to the experimental program to be discussed in the next section, because it demonstrates that detailed measurements carried out in a twodimensional unsteady flow have direct application to the three dimensional problem that is of technological interest. And it is clear that the experimental technique is greatly simplified in the twodimensional unsteady analogy and that a wider range of conditions may be covered. 


\section{EXPERIMENTAL STUDIES IN PROGRESS}

We are at present in the early stages of an extensive experimental program to investigate the detailed mechanism of shock induced enhancement of mixing and reaction in non-uniform gas streams. The process described earlier has been divided into two concurrent and relatively independent tasks, each of which concentrates on a critical portion of the physics. The first of these is being carried out in the Galcit 17-inch shock tube facility; the details of this facility are described by Liepmann et al (1962). The second investigation utilizes our unsteady combustion facility which is an outgrowth of several years of extensive research on combustion instability. The construction of this facility is described by Smith (1985) and by Smith \& Zukoski (1985).

The shock tube program builds upon the previous experimental work of Sturtevant and Haas, Hass (1983). The initial portion of our program is concerned with the interaction between a shock wave propagating through air and a cylindrical mass of gas. The aims are to obtain a quantitative measure of the distribution of helium in a thin section normal to the axis of the cylinder and, further, to obtain local measurements of the degree of molecular mixing which has taken place. This latter measurement is of great importance because it will be the first reliable indication of the extent to which the shock interaction is able to enhance the combustion chemistry. In each case, it is the aim to carry the measurements through times longer than those available from the sturtevant and Has data.

The shock tube mixing studies will be based on the fluorescence of biacetal, a dye which, when illuminated with radiation in the $v$ isible range, produces radiation in the visible range. This technique has been employed effectively, particularly by Epstein (1977). The dye will be added to the low density 888 which, in turn, is contained within a microfilm membrane until the shock impingement fractures it. After an accurately determined time delay, a pulse of light from a 3 - Joule dye laser, in the form of a thin sheet, will be passed normal to the helium cylinder. The resulting radiation will be measured using an intensified video camera and the data examined subsequently to find the distribution of helium. This experiment will be performed with a sequence of values of time delay in order to provide an accurate record of the development of mixing. The minimum thickness of the laser sheet which will provide a sufficiently strong signal is about $0.1 \mathrm{~cm}$. The video camera has an array of 244 by 388 pixels and this will limit the dimensions of the observed area also to about $0.1 \mathrm{~cm}$. Because the original scale of the helium region will be in the range of 2 to $5 \mathrm{~cm}$, the $0.1 \mathrm{~cm}$ resolution available will be quite adequate to study the gross mixing between the helium and air.

The question of molecular mixing is being addressed through a further development of the biacetyl technique. It is based upon the fact that, when appropriately excited benzene molecules collide with biacetyl molecules, a fraction of then transfer energy to the biacetyl molecules, leading them to phosphoresce. We shall employ this 
technique by placing the biacetyl dye in the belium, as before, and the benzene in the shock tube gas. After the shock wave has passed, the benzene will be excited by passing a sheet of pulsed laser light through the test region, the biacetyl dye will phosphoresce after collision with an excited benzene molecule, and the phosphorescence radiation will be recorded with the video camera.

A possible problem arises because the phospherescence radiation may be emitted over a period of milliseconds and an exposure $t$ ime of microseconds is desirable to stop the motion of the gas. The question is then whether the integrated radiation over the exposure time gives a sufficiently strong signal. Although this technique has been used successfully, Cheng (1978), it is recognized as higher risk than the basic mixing experiment. However, the importance of such results is such as to make reasonable compromises acceptable.

The second portion of the experimental research program is concerned with the details of combustion with in a vortex, carried out on a much larger physical scale and a longer time scale. This work is being performed utilizing our unsteady combustion facility shown in Fig. 12 in the configuration appropriate for the present experiments. A conventional blowdown supply system is used to furnish the combustible mixture from a $15.2 \mathrm{~cm}$. diameter plenum chamber, through a converging nozzle with a $9: 1$ contraction ratio, into a twodimensional, rectangular combustion chamber having a $15 \mathrm{~cm}$ by $7.6 \mathrm{~cm}$ cross section and a length of one meter. Gas will enter through an opening $2.5 \mathrm{~cm}$ by $7.6 \mathrm{~cm}$ in the top of the upstream wall. The velocity of the combustible gas mixture can be varied between 10 and $100 \mathrm{~m} / \mathrm{sec}$.

The combustion chamber permits access for pressure measurements, flow visualization, and optical measurements. The upper and lower walls are fabricated so that the inner steel liner of the combustor is cooled by water flowing inside an aluminum cooling jacket. The side walls are segmented and secured to the upper and lower walls by a window frame structure. Pycore glass walls are used for flow vizualization and optical experiments.

Velocity fluctuations will be forced at the inlet lip by producing large amplitude pressure fluctuations in the plenum chamber. These will be generated by a siren placed at the upstream end of the plenum chamber, adjusting the length of the plenum chamber to resonate with the desired acoust ic frequency. Part of the combustable mixture will pass through the siren, the rest through a bypass system. The amplitude of the oscillation will be controlled by varying the flow fraction through the siren. The system is designed to drive oscillations from 100 to $600 \mathrm{hz}$.

As a result of these velocity fluctuations, vortices are generated at the 1 ip of the downstream facing step, Hendricks(1986), and develop in the shear layer which separates the unburned flow from the recirculation zone. The individual vortices are ignited by the bot combustion products in the recirculation zone. The time scale for production and growth of these vortices will be affected by the size of the apparatus, the gas velocity, as well as the strength of the disturbance imposed to form each vortex, Sterling \& Zukoski (1987). 
In our system, we expect it to be in the range of 10 to 50 milliseconds. Although this is much longer than the time scale of the shock tube experiment, it does 1 imit the instrumentation and techniques which can be used in the observation. The exposure time for the image intensified video camera, with which we plan to observe the chemiluminescence of the burning gas, can be 88 small as a few microseconds but the framing rate of $60 \mathrm{hz}$ is too low to allow the development of a single vortex to be observed.

As a consequence, we have chosen to generate vortices periodically and to use phase averaging techniques which will permit determining the $t$ ime resolved properties of the flow. The experiment involves producing a train of vortices with a repetition rate of 100 to $500 \mathrm{hz}$, making measurements at a frequency of about $60 \mathrm{hz}$ which are timed to cover a complete cycle of the vortex. The complete experiment will take about one second and requires careful timing between exposure and the phase of vortex shedding. 


\section{CONCLUDING REMARKS}

It is clear that any augmentation of the rate of mixing between bydrogen fuel and air in a supersonic combustion ramjet offers welldefined advantages so far as engine performance and eng ine-airframe integration are concerned. The theory and technique of shock induced mixing enhancement, which has been introduced and discussed here, offers a very attractive possibility for significant reduction in the time (length) required for the mixing process. It has been shown, moreover, that a major portion of the demonstration of this concept may be carried out through time-dependent, two-dimensional studies in which the gas conditions corresponding to the proposed flight corridor may be reproduced to a considerable degree. We anticipate that the shock tube investigation of mixing and reaction, the study of unsteady combustion in vortices and, finally, experiments under conditions of steady flow, will confirm the degree to which this technique merits incorporation into engine development programs. 


\section{REFERENCES}

Cheng, W. R. (1978), "Turbulent Mixing in a Swirling Flow." Report No. 143. G8s Turbine and Plasma Dynamics Laboratory, Massachusetts Institute of Technology.

Edwards, C. I. W., Small, W. J., Weidner, J. P., and Johnston, P. J. (1975), "Studies of Scramjet/Airframe Integration Techniques for Eypersonic Aircraft." AIAA Paper 75-78.

Epstein, A. H. (1977), "Quantitative Density Visualization in a Transonic Rotor." ASME Journal of Power Engineering, pp. 460-475.

Evans, J. S., and Anderson, G. Y. (1975), "Supersonic Mixing and Combustion in Parallel Injection Flow Fields." Proceedings. AGARD Conference on Analytical and Numerical Methods for Investigation of Flow Fields with Chemical Reactions, Especislly Related to Combustion CP-164.

Evans, M. W., Harlow, F. H., and Meixner, B. D. (1962) "Interaction of Shock or Rarifaction with a Bubble." Physics of Eluids $\nabla .5$, No. 6, PP 651-656.

Ferri, A. (1968), "Review of SCRAMJET Propulsion Technology." J.Aircraft $\nabla .5$, No.1, 3-10.

Ferri, A. (1973), "Mixing-Controlled Combustion." Annual Reviews of Eluid Mechanics $\nabla$. 5, pp. 301-338.

Fleming, G. C.(1982), "Structure and Stability of Buoyant Diffusion Flames." Ph.D. Thesis, California Institute of Technology.

Gross, R. (1959), "Research on Supersonic Combustion." J. Amer. Rocket Society $\nabla .29, p 63$.

Gross, R. and Oppenheim, A. R. (1959), "Recent Advances in Gaseous Detonation." J. Amer. Rocket Society V. 29, pp 173-179.

Haas, J-F. L. (1983), "Interaction of Weak Shock Waves and Discrete Gas Inhomogeneities." Ph.D. Thesis California Institute of Technology.

Hendricks, G. J. (1986), "Two Mechanisms of Vorticity Generation in Combusting Flow Fields." PhD Thesis, California Institute of Technology.

Karagozian, A. R. and Marble, F. E. (1986), "Study of a Diffusion Flame in a Stretched Vortex". Combustion Science and Technology V. 45, PP 65-84.

Karagozian, A. R. and Manda, B. V. S.,(1987), "Flame Structure and Fuel Consumption in the Field of a Vortex Pair." To appear in Combustion Science and Technology. 
Kirkham, F. S., Hunt, J. L. (1977), "Hypersonic Transport Technology." Acta Astropautica Vo1. 4, 181-199.

L iepmann, H. W., Roshko, A., Coles, D., and Sturtevant, B. (1962), "A 17-inch Diameter Shock Tube for Studies in Rarified Gasdynamics." Rev. Sci. Instr. 7. 33. No. 6, pp. 625-631.

Marble, F. E. (1985), "Growth of a Diffusion Flame in the Field of a Vortex." Recent Advances in Aerospace Sciences Ed. C. Casci, Pp 395413.

Marble, F. E. and Hendricks, G. J. (1986a), "Behavior of a Diffusion Flame in a Flow Inducing a Pressure Gradient Along its Length." To appear in Proceedings, 21st International Symposium on Combustion.

Marble, F. E. and Hendricks, G. J. (1986b), "Distortion by Shock Impingement of Light Gas Regions Embedded in a Heavier Gas." Manuscript in preparation, unpublished.

McClinton, C. R. (1978), "Interaction Between Step Fuel Injectors on Opposite Walls in a Supersonic Combustor Model." NASA Technical Paper 1174.

Northam, G. B. and Anderson, G. Y. (1986), "Review of NASA/Lang ley Basic Research on Supersonic Combustion." AIAA Preprint 86-0159.

Nowak, R. J. and Relly, N. H. (1976), "Actively Cooled Airframe Structures for High Speed Flight." AIAA/ASME/SAE 17th Structures. Structural Dynamics and Materials Conference.

Picone, J. M. and Boris, J. P. (1983), "Vorticity Generation by Asymmetric Energy Deposition in a Gsseous Medium." Physics of Fluids V. 26, No. 2, pp 365-382.

Picone, J. M., Oran, E. S., Boris, J. P. and Young, T. R., Jr. (1983), "Theory of Vorticity Generation by Shock Wave and Flame Interaction." Progress in Astronaut ic s and Aeronautics $\nabla .94$, PP. 429-448.

Rudinger, G. (1958), "Shock Wave and Flame Interactions." Combustion and Propulsion Third AGARD Colloquium, PP. 153-182.

Rudinger, G. and Somers, I. (1960), "Behavior of Small Regions of Different Gases Carried in Accelerated Gas Flows." J. Eluid Mech. 7, 161-176.

Sma11, W. J., Weidner, J. P., and Johnston, P. J. (1974), "Nozzle Design and Analysis as Applied to a Bighly Integrated Research Airplane." NASA TM X-71972.

Smith, D. A. (1985), "An Experimental Study of Acoustically Excited, Vortex Driven, Combustion Instability Within a Rearward Facing Step Combustor." Ph.D. Thesis California Institute of Technology.

Smith, D. A. and Zukoski, E. E. (1985), "Combustion Instability Sustained by Vortex Combustion." 21st AIAA Joint Propulsion Conference. 
Sterling, J. D. and Zukoski, E. E. (1987), "Longitudinal Mode Instabilities in a Dump Combustor." AIAA 25th Aerospace Sciences Meeting, Jan 12-15, Reno.

Waltrup, P. J. (1986), "Liquid Fueled Supersonic Combustion Ramjets: A Research Perspective of the Past, Present and Future." AIAA 86-0158. 


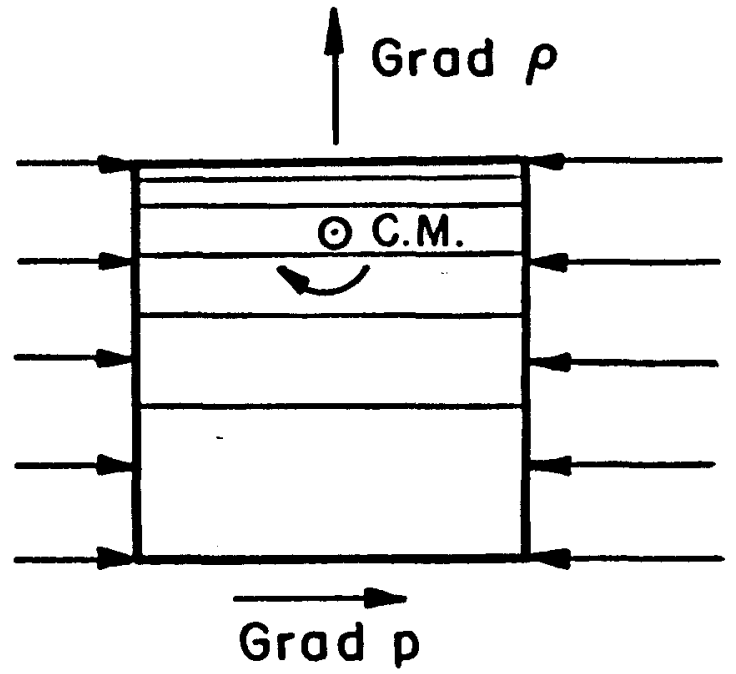

1. Vorticfty Produced by Interaction of Gradients in Pressure and Denstty 


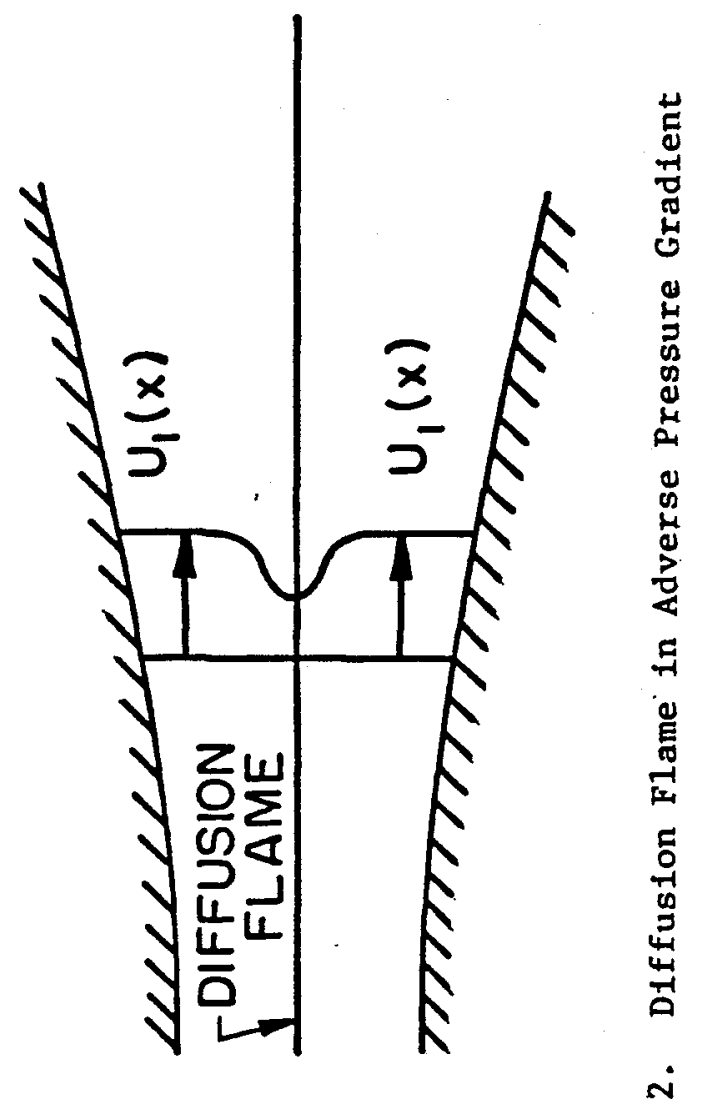




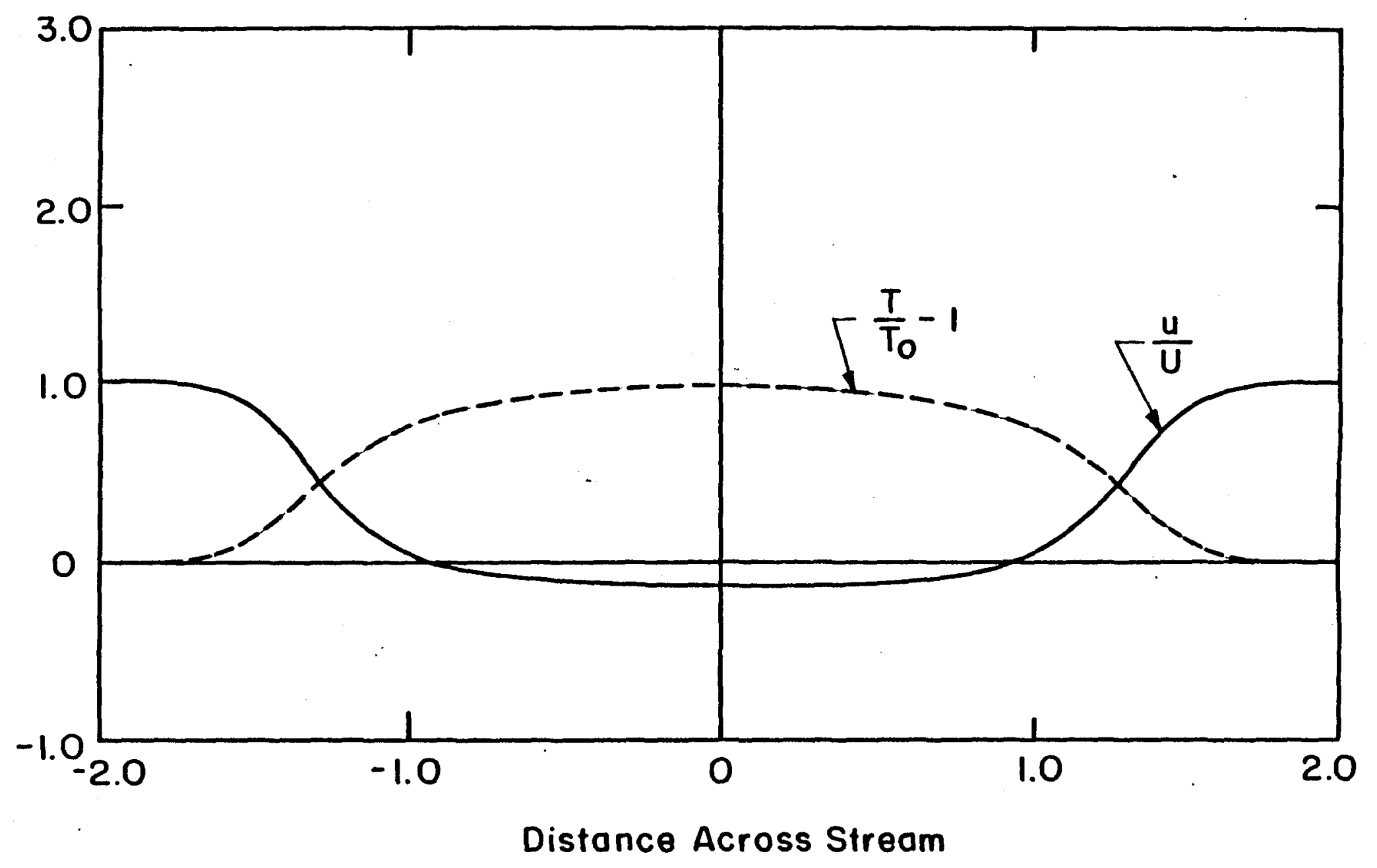

3. Backflow Induced in Diffusion Flame 


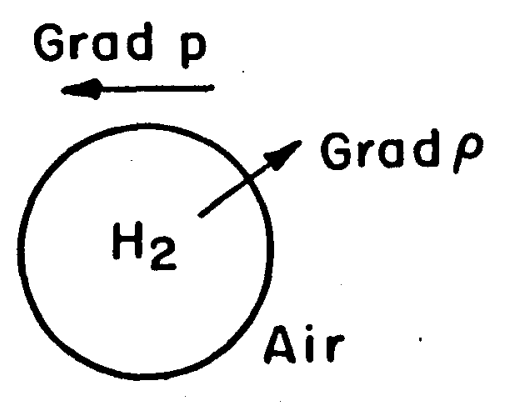

a.

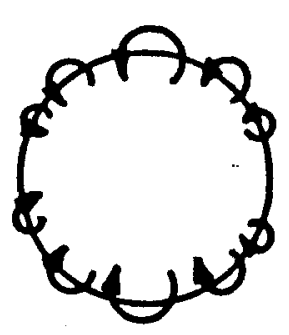

b.

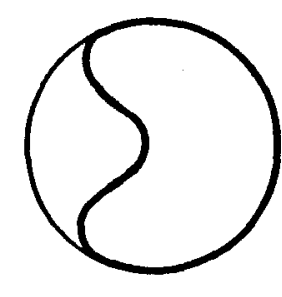

C.

4. Vorticity and Distortion Induced by Shock Passage over Hydrogen Cylinder in Air 


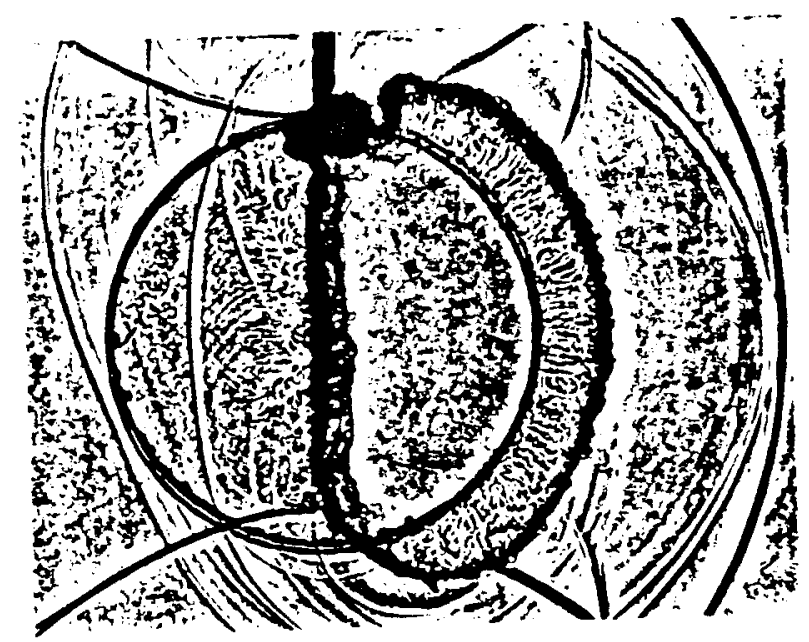

a) $0.132 \mathrm{~ms}$

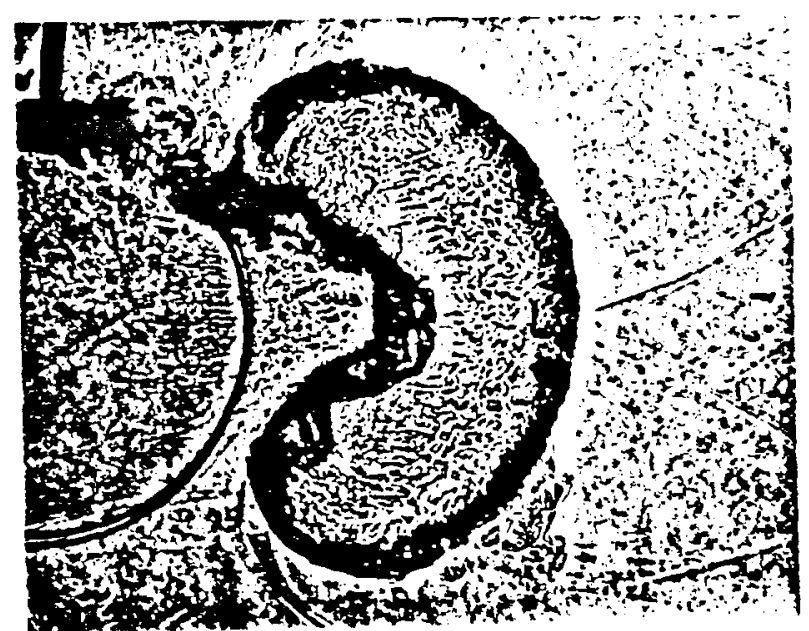

b) $0.378 \mathrm{~ms}$

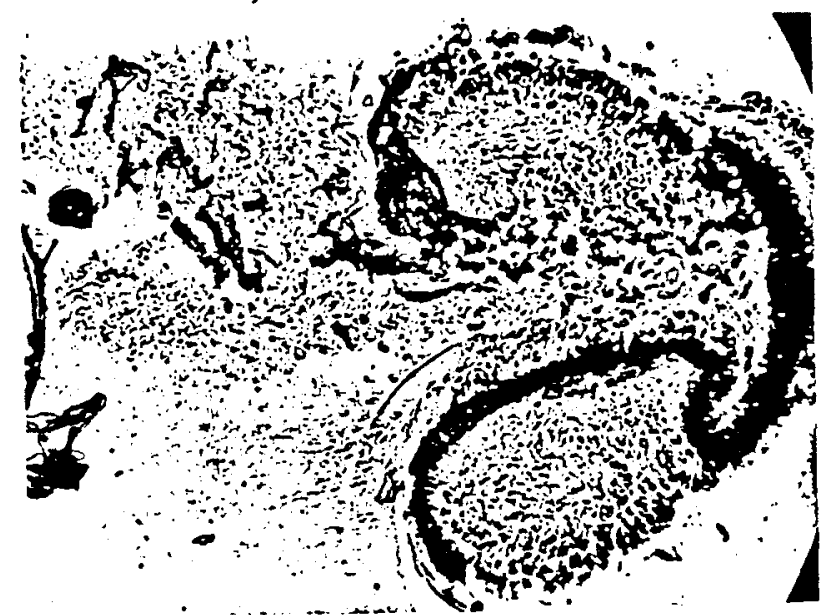

c) $0.724 \mathrm{~ms}$

5. Shadowgraphs of Helium Cylinder Distortion by Mach 1.22 Shock 

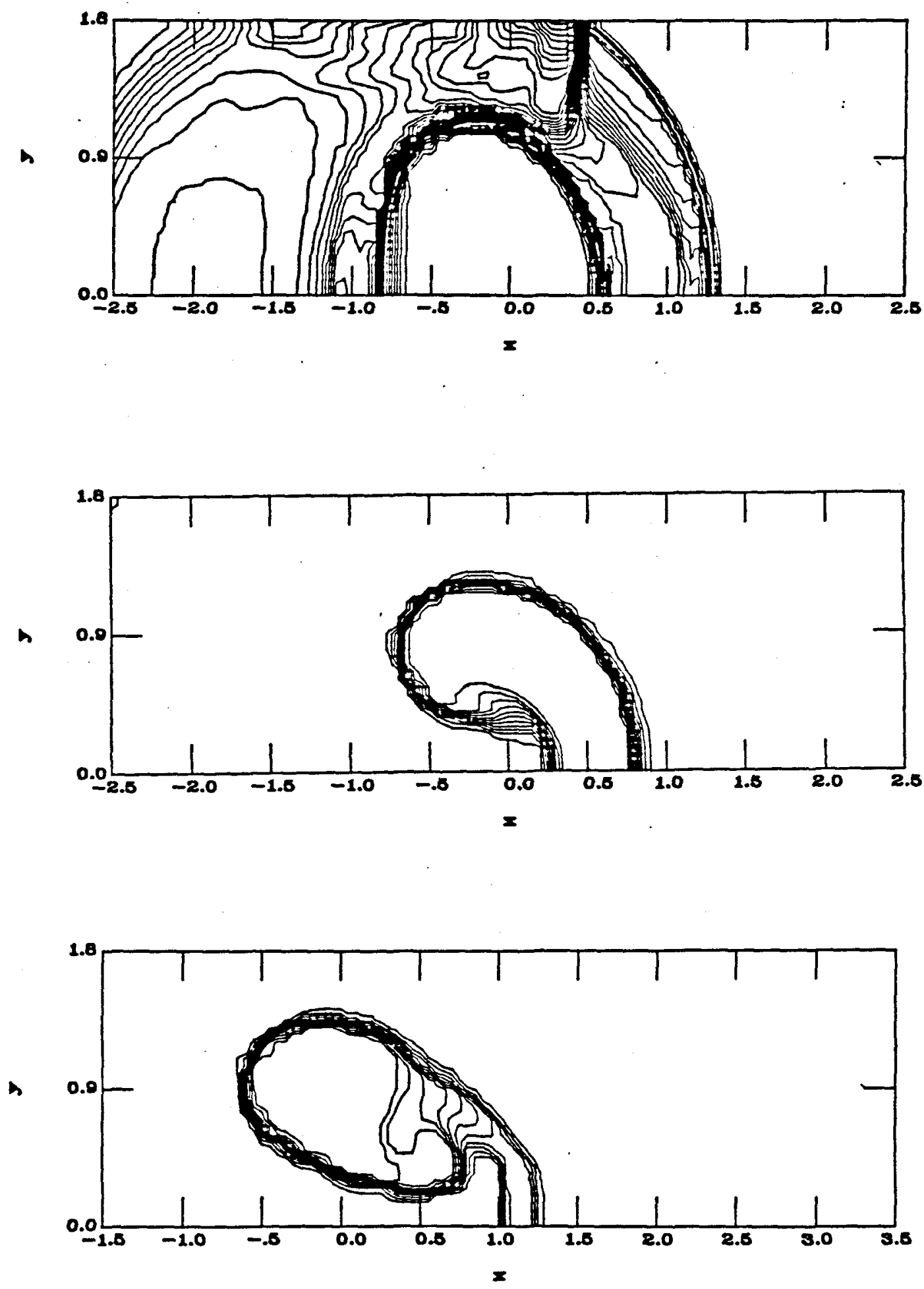

6. Computed Density Contours Corresponding to Shadowgraphs of Figure 5 . 


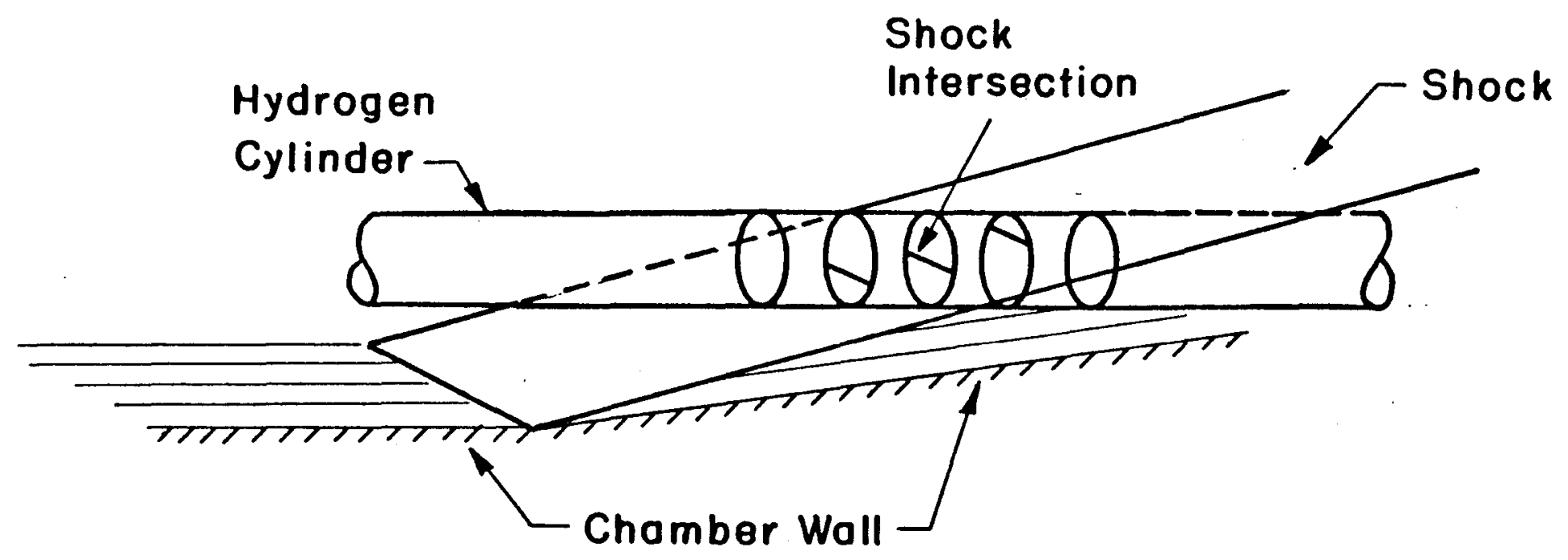

7. Schematic View of Oblique Shock Passage over Cylindrical Hydrogen Jet in Air 

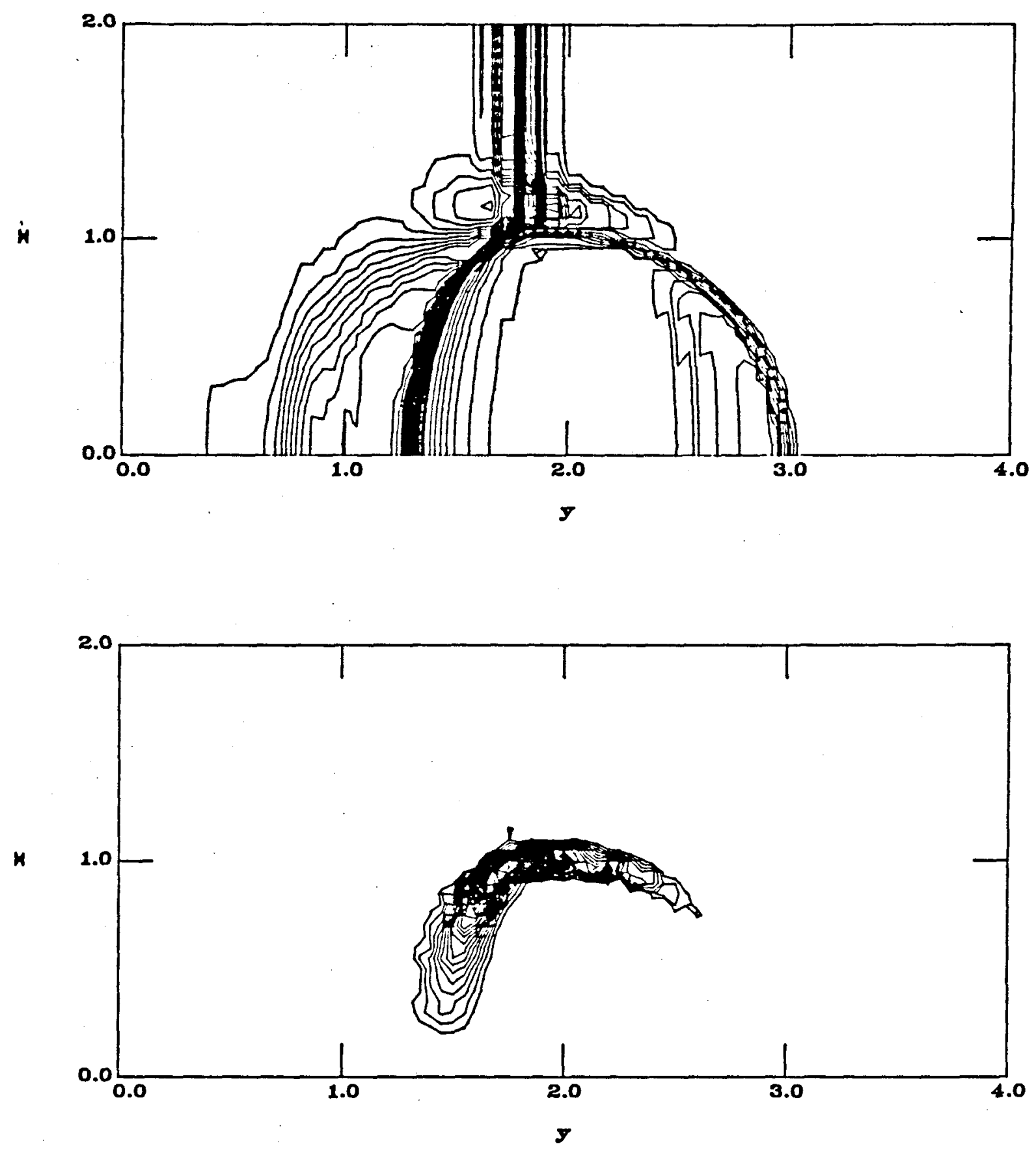

8. Density and Vorticity Distributions in Cylindrical Hydrogen Jet, Early Cross Section 

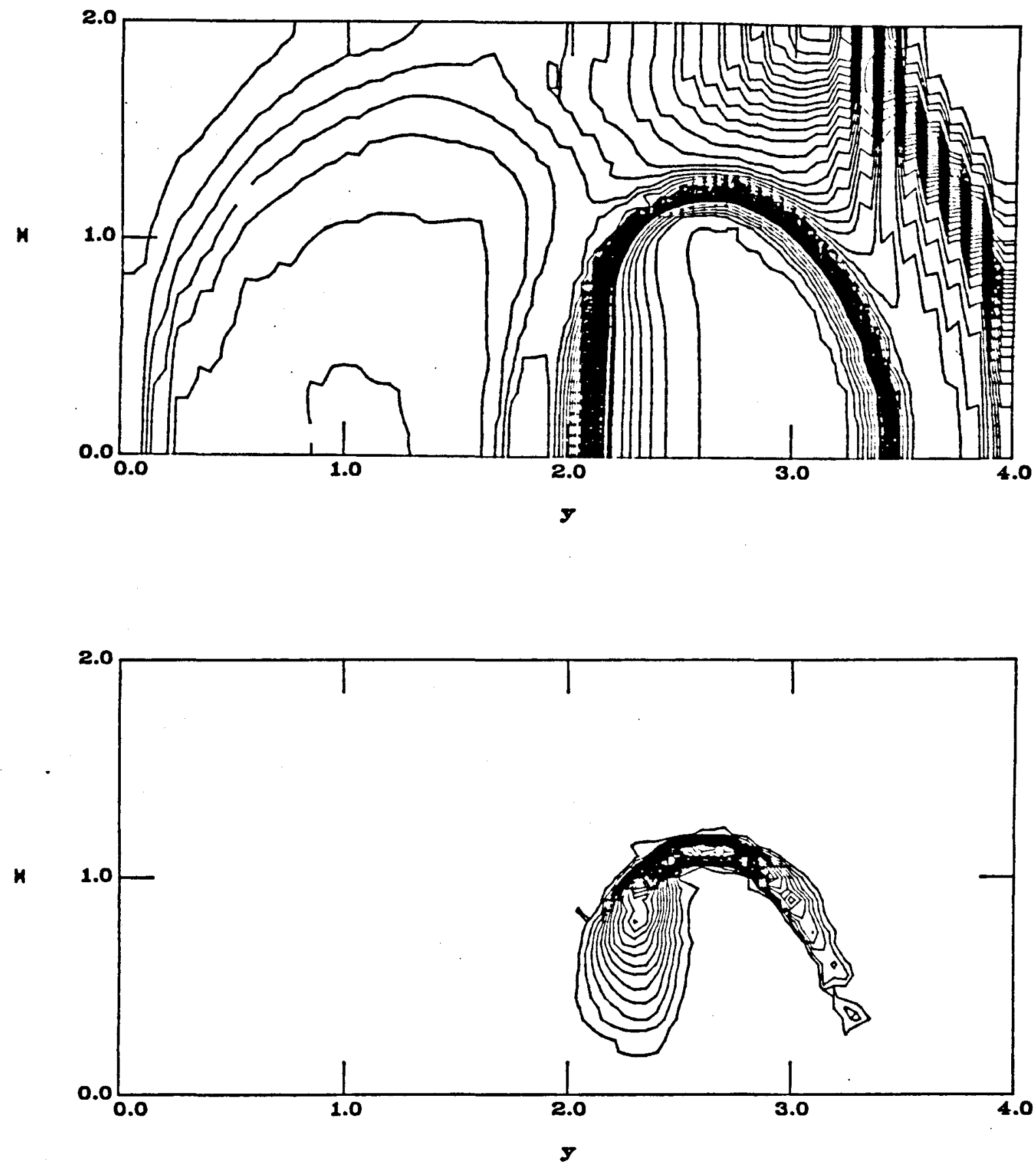

9. Density and Vorticity Distributions in Cylindrical Hydrogen Jet, Later Cross Section 

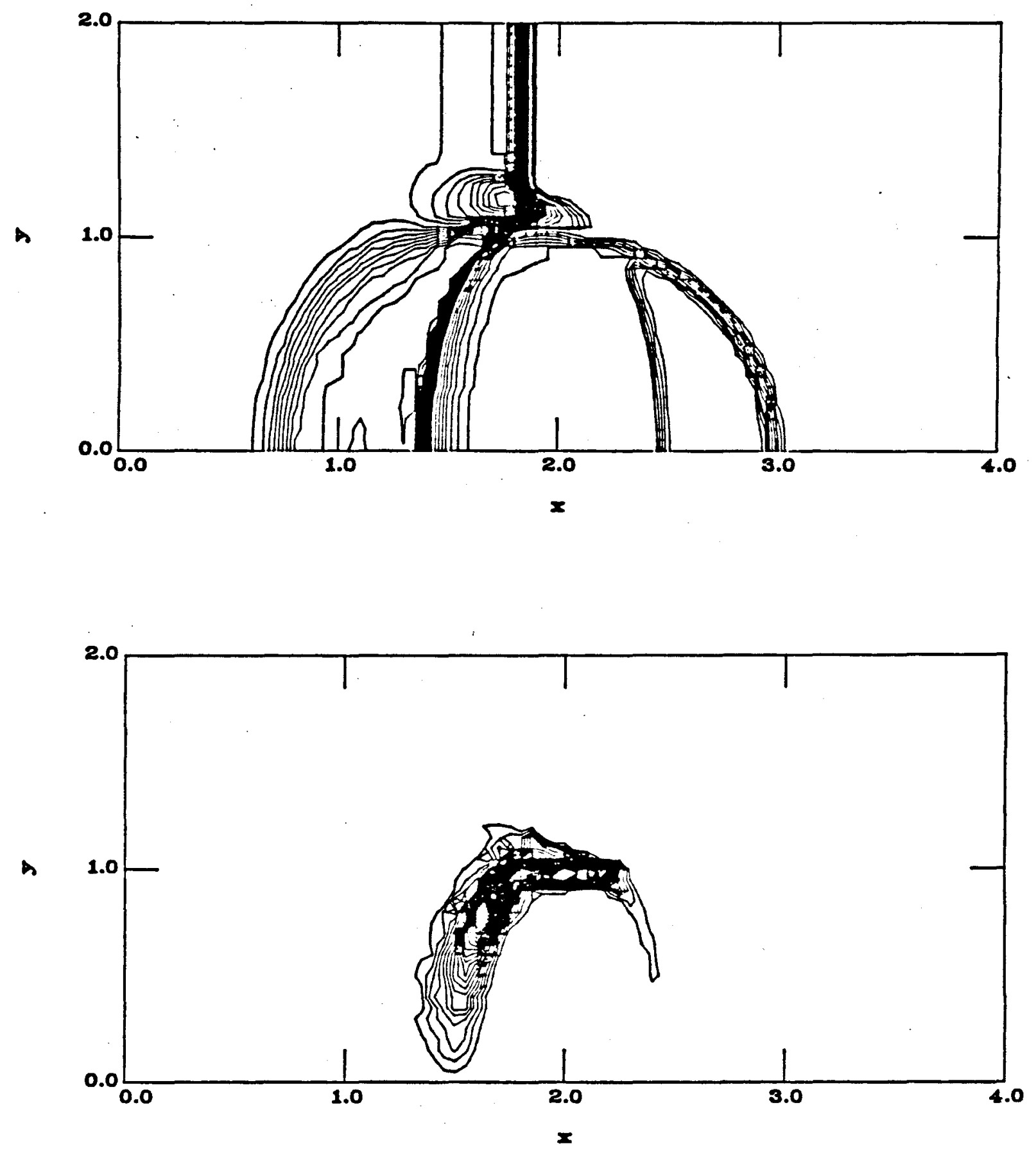

10. Two-Dimensional Computation of Density and Vorticity Corresponding to Three-Dimensional Calculations of Figure 8 

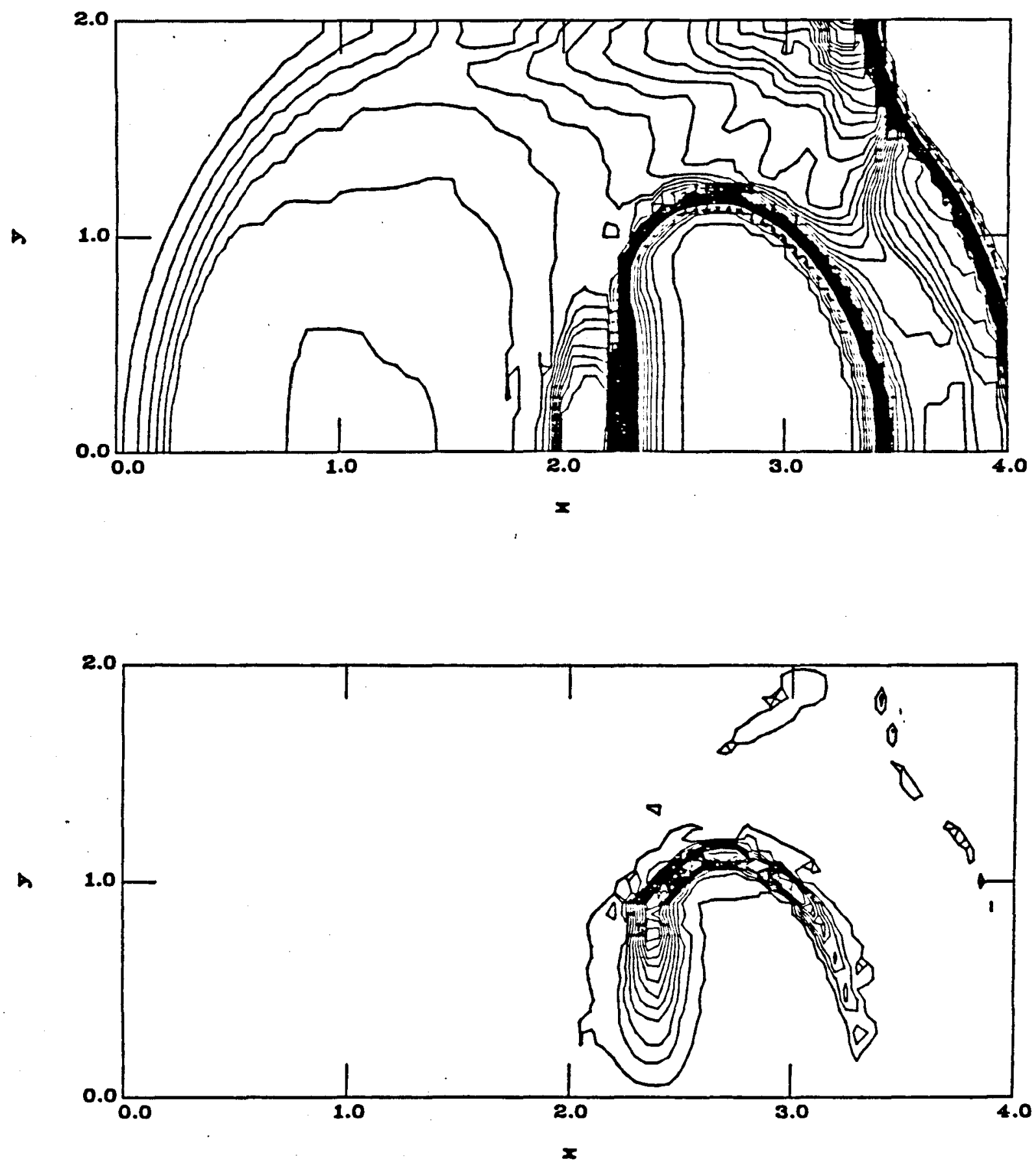

11. Two-Dimensional Computation of Density and Vorticity Corresponding to Three-Dimensional Calculations of Figure 9 


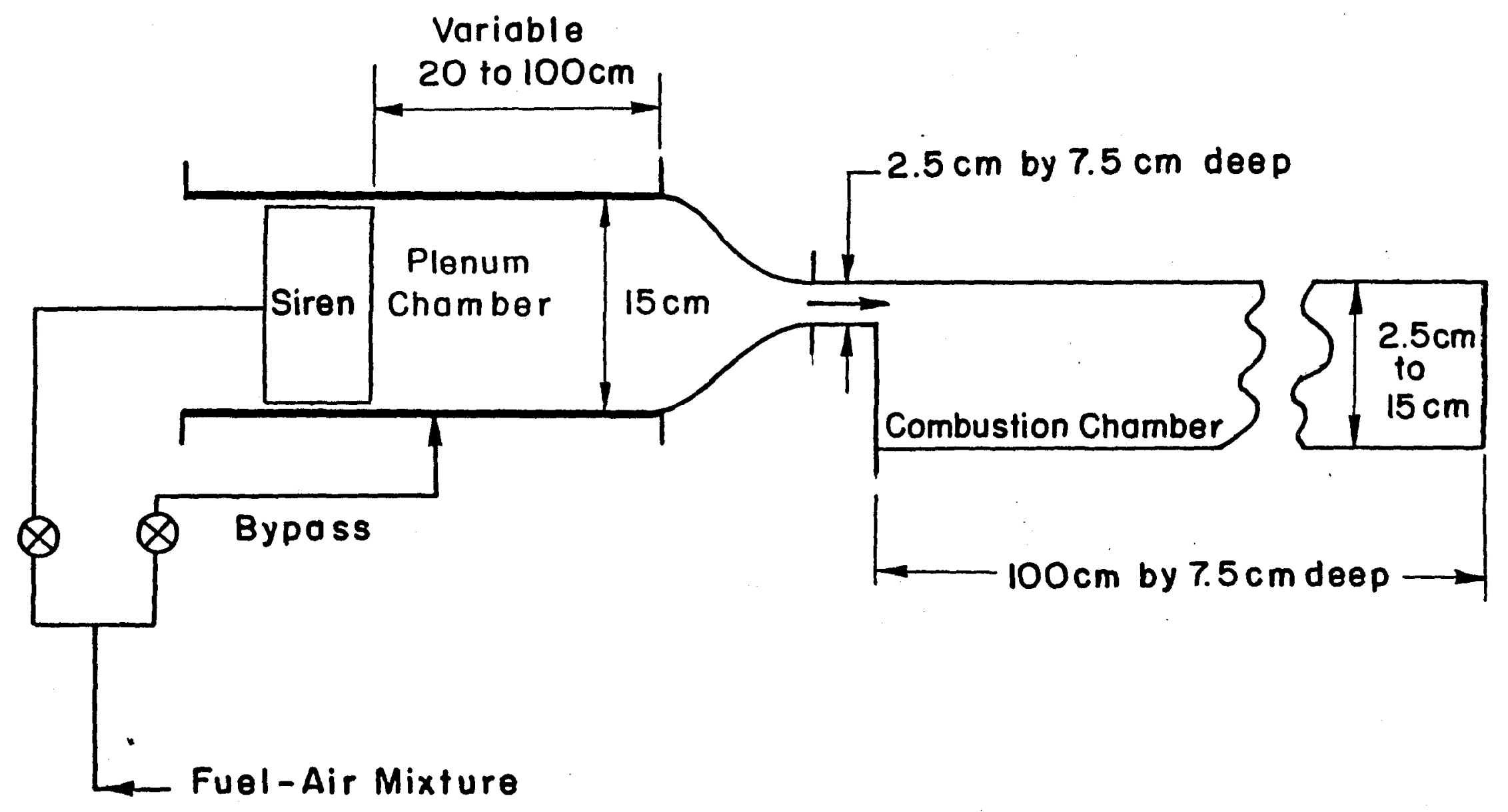

12. Schematic View of Unsteady Combustion Facility for Study of Vortex Combustion 